\title{
Formulation and Evaluation of Chitosan-Chondroitin Sulphate Based Nasal Inserts for Zolmitriptan
}

\author{
Kirandeep Kaur and Gurpreet Kaur \\ Department of Pharmaceutical Sciences and Drug Research, Punjabi University, Patiala, Punjab 147002, India \\ Correspondence should be addressed to Gurpreet Kaur; kaurgpt@gmail.com
}

Received 25 April 2013; Accepted 26 August 2013

Academic Editor: Shirui Mao

Copyright (C) 2013 K. Kaur and G. Kaur. This is an open access article distributed under the Creative Commons Attribution License, which permits unrestricted use, distribution, and reproduction in any medium, provided the original work is properly cited.

\begin{abstract}
Bioadhesive nasal dosage forms are an attractive method for overcoming rapid mucociliary clearance transport in the nose and for delivering the drug directly to brain. The present study was designed to formulate chondroitin sulphate (CS) and chitosan $(\mathrm{CH})$ nasal inserts employing zolmitriptan, an antimigraine drug. The interpolymer complexes (IPC) formed between $-\mathrm{COO}^{-}$and $-\mathrm{OSO}_{3}{ }^{-}$groups of $\mathrm{CS}$ and $-\mathrm{NH}_{3}{ }^{+}$group of $\mathrm{CH}$ were characterized by infrared spectroscopy (IR), differential scanning analysis (DSC), and zeta potential studies. The unloaded and loaded nasal inserts were evaluated for water uptake studies, and bioadhesive strength studies, scanning electron microscopic studies (SEM). The in vitro drug release and in situ permeation studies were carried out on loaded nasal inserts. The DSC and IR studies confirmed the formation of a complex between the two polymers. The results indicated that the formulation $\mathrm{F} 1(\mathrm{CH}: \mathrm{CS} ; 30: 70)$ was demonstrating the highest bioadhesive strength and zeta potential. The presence of porous structure in the nasal inserts was confirmed by the SEM analysis. Further, in vitro and in situ release studies demonstrated that formulations F9 and F11 (drug : polymer; $1: 10$ ) were releasing $90 \%$ and $98 \%$ zolmitriptan over a period of $8 \mathrm{~h}$. It can be concluded that nasal inserts formulated from chitosan-chondroitin sulphate (CH-CS) interpolymer complex (IPC) can be used for delivery of antimigraine drug to brain.
\end{abstract}

\section{Introduction}

Migraine is one of the most common headache disorders that affect $17-18 \%$ of the female and $6 \%$ of the male population. It is characterized by episodic unilateral headache attacks that are accompanied by nausea and/or photo- and phonophobia [1]. Current migraine treatment is divided into acute care and daily preventive medication. The acute treatment includes administration of simple analgesics like aspirin and NSAIDs; combination analgesics like caffeine with aspirin, acetaminophen, or caffeine; ergot alkaloids like ergotamine tartrate, dihydroergotamine, and triptans like sumatriptan, zolmitriptan; and naratriptan [2]. Migraine is frequently accompanied with severe nausea, poor gastrointestinal (GI) absorption, or vomiting; as a result oral administration of drugs becomes difficult [3]. The nasal route of administration offers an attractive alternative to the oral route for drug delivery. Intranasal drugs are transported along the olfactory sensory neurons to yield significant concentrations in the cerebrospinal fluids [4]. Intranasal route is noninvasive and painless, does not require sterility regulations, and is readily administered by the patient. However, the nasal cavity presents a number of limitations for drug absorption including enzymatic degradation, low intrinsic permeability for hydrophilic drugs, proteins, and peptides, and rapid mucociliary clearance. Therefore, in order to overcome these barriers a number of strategies can be applied by modifying three components: the drug, delivery carrier, and administration device [5]. Nasal inserts, a promising drug delivery system, are prepared by lyophilization of polymers. They consist of a sponge-like hydrophilic polymer matrix, in which the drug is embedded. They allow easy dosing with a high potential for systemic administration that helps prevent hepatic first pass metabolism. When the nasal insert comes into contact with the highly vascularized nasal mucosa, it absorbs water and swells. The polymer gel releases the active ingredient in a controlled manner [6]. Zolmitriptan is a second generation triptan prescribed for patients with migraine attacks, with or without aura and cluster headaches. It selectively acts on serotonin receptors and is very effective 
in reducing migraine symptoms, including pain, nausea, and photo- or phonophobia [7]. It is currently available as a conventional tablet, an oral disintegrating tablet, and a nasal spray. However, the bioavailability of nasal spray is very low due to rapid clearance of the drug from nasal cavity [8]. Therefore the emphasis now is to develop bioadhesive nasal delivery systems. Natural polysaccharides are generally regarded as safe (GRAS); they show interesting biological properties, including biocompatibility, biodegradability, and bioadhesion. A combination of polymers has been found to demonstrate better functional properties as compared to a single polymer. Further, tailor-made multiple polymers or interpolymer complexes have been exploited in the field of drug delivery to meet specific demands [9]. Therefore, in this study chitosan- (CH-) chondroitin sulphate (CS) interpolymer complexes (IPC) were employed for the formulation of bioadhesive nasal inserts. $\mathrm{CH}$ is a cationic polymer obtained by deacetylation of chitin, the polymer forming the exoskeleton of crustaceans. It has the capacity to open tight junctions and thus increases paracellular transport in the epithelial membrane, possesses nontoxicity, and low immunogenicity $[10,11]$. Chondroitin sulphate (CS) is found in bone, cartilage, and connective tissue and composed of acetyl galactosamine and glucuronic acid residues alternately linked to each other by the $\beta 1-4$ and $\beta 1-3$ bonds, respectively. CS is an anionic mucopolysaccharide, which is able to form ionic complexes with positively charged substance [12]. CH-CS IPC were characterized by spectral and thermal analysis. The freeze-dried inserts were evaluated for their morphological characteristics, swelling studies, zeta potential, bioadhesive potential, and in vitro release and in situ permeation studies.

\section{Experimental}

2.1. Materials. Chitosan was purchased from India Sea Foods, Cochin. Chondroitin sulphate was received as a gift sample from Panacea Biotech, Lalru. Zolmitriptan was purchased from Molekula Life Sciences Limited, Hyderabad. All other reagents and chemicals were of analytical grade and were used as such.

2.2. Preparation of $\mathrm{CH}$-CS IPC. CH-CS IPC were prepared as reported by Luppi et al. [13]. CH and CS were dissolved separately in $25 \mathrm{~mL}$ of acetate buffer ( $\mathrm{pH} 5.0$ ) producing a $2 \%$ w/v of polymer solution. The $\mathrm{CH}$ solution was then added to CS solution in various molar ratios $(30: 70,40: 60,50: 50$, $60: 40$, and $70: 30 ; \mathrm{CH}: \mathrm{CS}$ ) and stirred at room temperature for $24 \mathrm{~h}$. The precipitates were separated by centrifugation (Remi Cooling Centrifuge, India) at 10,000 rpm for $10 \mathrm{~min}$, washed with deionised water, and dried. The practical yield of the IPC was calculated employing the following equation:

$$
\text { percent yield }(\%)=\frac{W_{0}}{W_{t}} \times 100
$$

where $W_{0}=$ weight of IPC obtained and $W_{t}=$ total weight of polymers taken.
TABLE 1: Composition and properties of unloaded nasal inserts.

\begin{tabular}{lcccc}
\hline $\begin{array}{l}\text { Formulation } \\
\text { code }\end{array}$ & $\begin{array}{c}\text { Composition } \\
(\mathrm{CH}: \mathrm{CS})\end{array}$ & Yield (\%) & $\begin{array}{c}\text { Viscosity } \\
(\mathrm{cP})\end{array}$ & $\begin{array}{c}\text { Water } \\
\text { uptake }\end{array}$ \\
\hline F1 & $30: 70$ & $33.68 \pm 4.23$ & $1.0 \pm 0.0$ & $2.88 \pm 0.24$ \\
F2 & $40: 60$ & $52.45 \pm 6.09$ & $1.0 \pm 0.0$ & $2.07 \pm 0.22$ \\
F3 & $50: 50$ & $55.22 \pm 6.78$ & $1.0 \pm 0.0$ & $1.65 \pm 0.15$ \\
F4 & $60: 40$ & $49.09 \pm 6.32$ & $11.0 \pm 1.0$ & $3.98 \pm 0.25$ \\
F5 & $70: 30$ & $35.35 \pm 4.56$ & $16.5 \pm 1.5$ & $4.52 \pm 0.56$ \\
\hline
\end{tabular}

\subsection{Characterization of IPC}

2.3.1. Attenuated Total Reflectance-Infrared (ATR-IR) Studies. The powdered polymers were characterized using ATR spectroscopy (ALPHA-E Bruker, Germany). The samples were scanned in the absorbance in the range from 4000 to $600 \mathrm{~cm}^{-1}$.

2.3.2. Differential Scanning Calorimetric Studies (DSC). DSC measurements were performed on powdered samples of pure $\mathrm{CH}$ and $\mathrm{CS}$ and their IPC in different ratios at a heating rate of $10^{\circ} \mathrm{C} / \mathrm{min}$ (Mettler Toledo $812 \mathrm{E}$, Switzerland) in the range from 30 to $400^{\circ} \mathrm{C}$.

2.3.3. Zeta Potential. Zeta potential $(\zeta)$ was measured using a Zetasizer (Malvern Instruments Ltd., Malvern, UK). The zeta potential measurements were performed by using an aqueous dip cell in an automatic mode. Samples were diluted in ultrapurified water and placed in a clear disposable zeta cell, with the cell position being adjusted.

2.3.4. Viscosity Measurements of $\mathrm{CH}$-CS IPC. The viscosity of the supernatants obtained after mixing solutions of $\mathrm{CH}$ with CS solutions in different ratios was determined on Brookfield viscometer spindle 00 (Brookfield viscometer LVDV1, Bruker, UK).

2.4. Preparation of Nasal Insert. The precipitates of $\mathrm{CH}$ and CS IPC were homogenized for $5 \mathrm{~min}$, suspended again in deionised water, and finally freeze-dried (Allied Frost Macflow Engineering, India) in microcentrifuge tubes, obtaining unloaded solid inserts (Table 1). Mannitol was added to increase the mechanical strength of nasal inserts in the ratio of complex/mannitol of $9: 1 ; \mathrm{w} / \mathrm{w}$. Drug loaded nasal inserts were prepared by adding zolmitriptan in the doses of $2.5 \mathrm{mg}$ and $5 \mathrm{mg}$ to $50 \mathrm{mg}$ (1:20 and 1:10 ratios, resp.) of different complex/mannitol mixtures $(9: 1 ; \mathrm{w} / \mathrm{w})$ obtaining ten different inserts (Table 2 ). The unloaded inserts were evaluated for the water uptake studies and bioadhesive strength studies and the loaded inserts were evaluated for in vitro drug release and in situ permeation studies.

\subsection{Evaluation of Nasal Inserts}

2.5.1. Water Uptake Studies. Accurately weighed unloaded nasal inserts $(50 \mathrm{mg})$ containing different concentrations of polymers $\mathrm{CH}$ and CS were placed on filter paper soaked in 
TABLE 2: Composition of drug loaded nasal inserts.

\begin{tabular}{lccc}
\hline $\begin{array}{l}\text { Formulation } \\
\text { code }\end{array}$ & $\begin{array}{c}\text { Composition } \\
(\mathrm{CH}: \mathrm{CS})\end{array}$ & Drug : polymer & $\begin{array}{c}\text { Content } \\
\text { uniformity } \\
(\%)\end{array}$ \\
\hline F6 & $30: 70$ & $1: 20$ & $98.13 \pm 0.77$ \\
F7 & $30: 70$ & $1: 10$ & $96.23 \pm 0.80$ \\
F8 & $40: 60$ & $1: 20$ & $97.44 \pm 0.70$ \\
F9 & $40: 60$ & $1: 10$ & $98.73 \pm 0.83$ \\
F10 & $50: 50$ & $1: 20$ & $97.21 \pm 1.25$ \\
F11 & $50: 50$ & $1: 10$ & $97.36 \pm 0.96$ \\
F12 & $60: 40$ & $1: 20$ & $98.71 \pm 1.56$ \\
F13 & $60: 40$ & $1: 10$ & $97.36 \pm 1.45$ \\
F14 & $70: 30$ & $1: 20$ & $98.55 \pm 0.98$ \\
F15 & $70: 30$ & $1: 10$ & $96.32 \pm 1.65$ \\
\hline
\end{tabular}

phosphate buffer $\mathrm{pH} 5.5$ and positioned on top of a sponge previously soaked in the hydration medium and placed in a petri plate filled with the same buffer to the height of $0.5 \mathrm{~cm}$. The water uptake by the insert was calculated [13]. The swelling behavior of the sponge was calculated using the following equation:

$$
P_{s}=\frac{\left(W_{s}-W_{i}\right)}{W_{i}} \times 100,
$$

where $P_{s}$ is percent swelling, $W_{s}$ is weight of the swollen insert at time " $t$ ", and $W_{i}$ is initial weight of the insert.

2.5.2. Measurement of Bioadhesive Studies. The in vitro bioadhesive strength was evaluated using texture analyzer equipment equipped with a $50 \mathrm{Kg}$ load cell (TA.XT Plus, Stable Micro Systems, UK). Excised nasal mucosa of an adult goat was used for the measurement of bioadhesive strength. It was obtained from a local slaughter house and placed in aerated saline solution at $4^{\circ} \mathrm{C}$ until used [14]. The nasal insert was mounted securely in place on a cylindrical probe (using double-sided adhesive tape) which was fixed to the mobile arm of the texture analyzer. The nasal mucosa was placed to the lower arm. The cylindrical probe with the membrane attached to its base was lowered at a speed of $0.5 \mathrm{~mm} / \mathrm{s}$ at a force of $1 \mathrm{~N}$ for a contact time of $30 \mathrm{~s}$. It was then withdrawn at a rate of $0.5 \mathrm{~mm} / \mathrm{s}$ to a distance of $10 \mathrm{~mm}$. The mucoadhesive performance of the samples was determined by measuring the resistance to the withdrawal of the probe (maximum detachment force) reflecting the mucoadhesion characterization of the polymeric formulations with nasal mucosa. At least three repetitions were obtained for each measurement.

2.5.3. Scanning Electron Microscopy (SEM). A very small amount of the sample was dropped on the sample holder with double-sided tape, extra powder was removed and coated for $70 \mathrm{~s}$ under an argon atmosphere with gold coating, and then the powdered samples on the SEM grid were allowed to airdry for $10 \mathrm{~min}$. Images were taken at different magnifications using SEM (Jeol JSM-6610 LV, USA) machine.
2.5.4. Drug Content Uniformity. Nasal inserts were weighed individually and the drug was extracted in methanol. The drug content was determined by filtering the solution through $0.4 \mu \mathrm{m}$ membrane. The drug content was analyzed by measuring absorbance spectrophotometrically (Beckman DU640B spectrophotometer, USA) at $225 \mathrm{~nm}$.

2.5.5. In Vitro Drug Release Studies. In vitro drug release studies were carried out in phosphate buffer $\mathrm{pH}$ 5.5. using USP XXX-NF XXV (Apparatus-1-Basket type) dissolution apparatus (Tab Machines, India), where $450 \mathrm{~mL}$ of phosphate buffer $\mathrm{pH} 5.5$ was used as dissolution media maintained at $37^{\circ} \mathrm{C} \pm 0.5^{\circ} \mathrm{C}$ at $50 \mathrm{rpm}$. Drug loaded inserts $(2.5 \mathrm{mg}$ and $5 \mathrm{mg}$ ) were placed in the dissolution vessel. Five $\mathrm{mL}$ of aliquot samples was withdrawn at 1, 2, 3, 4, 5, 6, 7, and $8 \mathrm{~h}$ and replaced with fresh media. Samples were then filtered through a $0.45 \mu \mathrm{m}$ filter and analyzed spectrophotometrically at $225 \mathrm{~nm}$.

2.5.6. In Situ Permeation Studies. In situ release study of loaded nasal insert was carried out by using Keshary-Chien Franz diffusion cell. The formulations containing $2.5 \mathrm{mg}$ and $5 \mathrm{mg}$ doses of zolmitriptan were placed in donor compartment and freshly prepared phosphate buffer $\mathrm{pH} 5.5$ in the receptor compartment. The nasal mucosa was placed between donor and receptor compartment. The whole assembly was placed on the thermostatically controlled $\left(37^{\circ} \mathrm{C} \pm 0.5^{\circ} \mathrm{C}\right)$ magnetic stirrer. One $\mathrm{mL}$ of sample was withdrawn at predetermined time interval of $1 \mathrm{~h}$ for a period of $8 \mathrm{~h}$ and the same volume of fresh medium was replaced. The withdrawn samples were analyzed by UV spectrophotometer at $225 \mathrm{~nm}$ using a buffer as a blank.

2.6. Statistical Analysis. In order to determine statistical significance, ANOVA was used and the differences were considered to be significant for values of $P<0.05$.

\section{Results and Discussion}

3.1. Characterization of IPC. The percent yield of IPC obtained after complexation of $\mathrm{CH}$ and $\mathrm{CS}$ are depicted in Table 1. It was observed that maximum yields was obtained when $\mathrm{CH}$ and $\mathrm{CS}$ were present in the ratio of $50: 50$. The ATR-IR spectra of $\mathrm{CH}, \mathrm{CS}$ and the IPC formed by interaction of various ratios of $\mathrm{CH}$ and $\mathrm{CS}$ showed characteristic bands (Figure 1). It is evident from Figure 1(a) that $\mathrm{CH}$ powder ( $85 \%$ deacetylation) exhibited a peak at $1560 \mathrm{~cm}^{-1}$ indicating presence of $-\mathrm{NH}_{3}{ }^{+}$ions. The peak at $1422 \mathrm{~cm}^{-1}$ (Figure 1(a)), suggesting presence of $-\mathrm{COO}^{-}$ions, could have arisen due to $15 \%$ acetylation of $\mathrm{CH}$ powder. The IR spectrum of CS is represented in Figure 1(b). The peaks observed at $1646 \mathrm{~cm}^{-1}$, $1416 \mathrm{~cm}^{-1}$, and $1130 \mathrm{~cm}^{-1}$ can be assigned, respectively, to $-\mathrm{CONH}_{2},-\mathrm{COO}^{-}$, and $-\mathrm{OSO}_{3}{ }^{-}$groups (Kemp, 1991). The CH-CS IPC displayed peaks in the ranges of 1400$1410 \mathrm{~cm}^{-1}$ and $1550-1560 \mathrm{~cm}^{-1}$ suggesting the formation of complex between $-\mathrm{NH}_{3}{ }^{+}$and $-\mathrm{COO}^{-}$groups of $\mathrm{CH}$ and CS, respectively (Figures 1(c), 1(d), 1(e), 1(f), and 1(g)). 


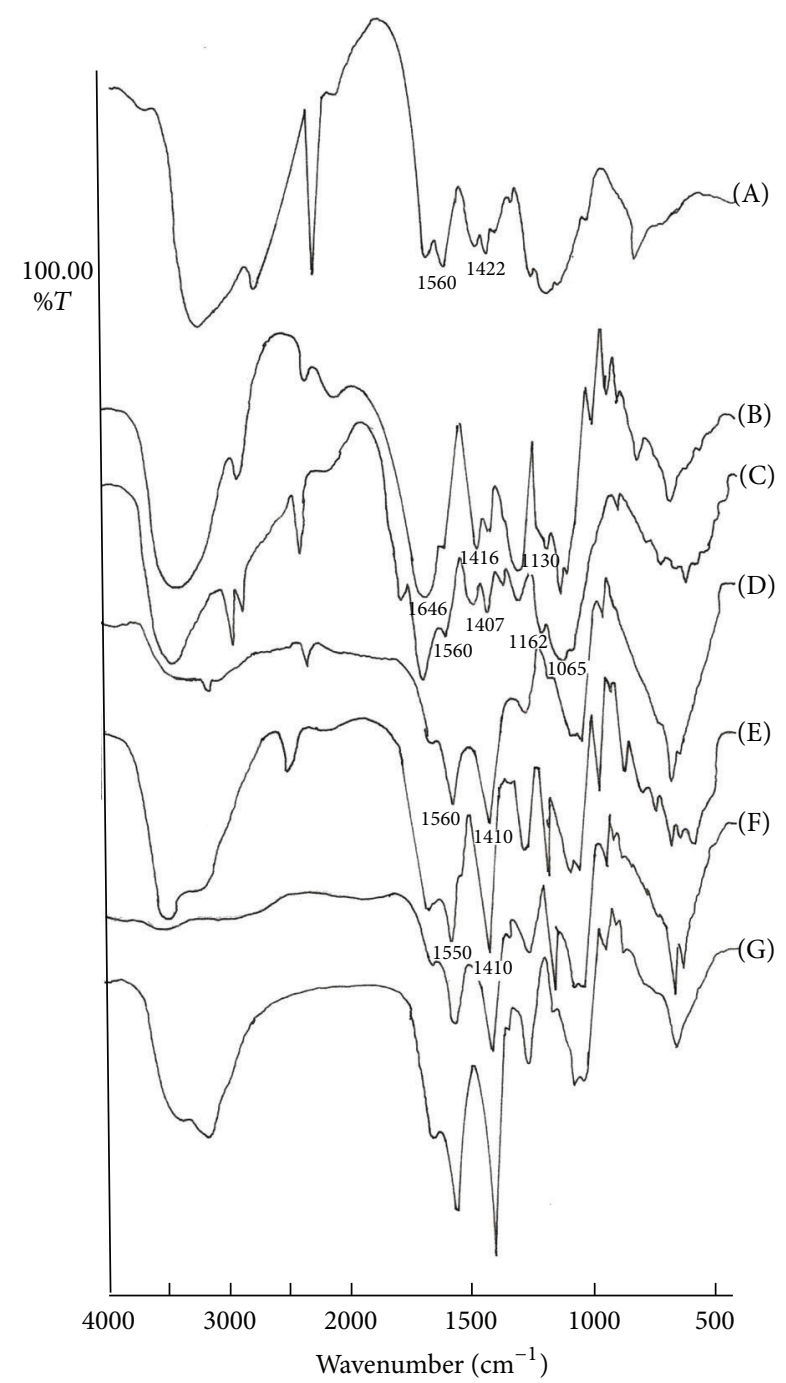

FIgURE 1: IR spectra of $\mathrm{CH}(\mathrm{A}), \mathrm{CS}(\mathrm{B})$, and IPCs in the ratio of $\mathrm{CH}: \mathrm{CS},(\mathrm{C}) 70: 30$ (D) $60: 40$ (E) $50: 50$ (F) $40: 60$, and (G) $30: 70$.

Further, the existence of a broad band at $1065 \mathrm{~cm}^{-1}$ with a shoulder at $1162 \mathrm{~cm}^{-1}$ in the IR spectrograph suggested the interaction between $-\mathrm{NH}_{3}{ }^{+}$and $-\mathrm{OSO}_{3}{ }^{-}$groups $[15,16]$. Figure 2 depicts DSC thermograms of $\mathrm{CH}$ and $\mathrm{CS}$ which indicated one endothermic and one exothermic transition. It is evident from Figure 2(a) that $\mathrm{CH}$ powder exhibited one endothermic and one exothermic transition each at, respectively, $80.22^{\circ} \mathrm{C}$ and $311.30^{\circ} \mathrm{C}$. The DSC thermogram of CS revealed one endotherm at $97^{\circ} \mathrm{C}$ and one exotherm at $239.69^{\circ} \mathrm{C}$. The thermograms of IPC films containing any ratio of $\mathrm{CH}$ : $\mathrm{CS}$ exhibited the first endothermic transition with peak temperature ranging from 90 to $110^{\circ} \mathrm{C}$. This was followed by a second endothermic transition with peak temperature ranging between 190 and $205^{\circ} \mathrm{C}$ (Figures 2(c)$2(\mathrm{~g}))$. The first broad endotherm can be suggested to have arisen due to evaporation of water from the IPC. The second endothermic transition occurring in the temperature range of $190-205^{\circ} \mathrm{C}$ was found to differ in the $\Delta H$ value amongst films containing different ratios of $\mathrm{CH}$ : CS. This suggested that

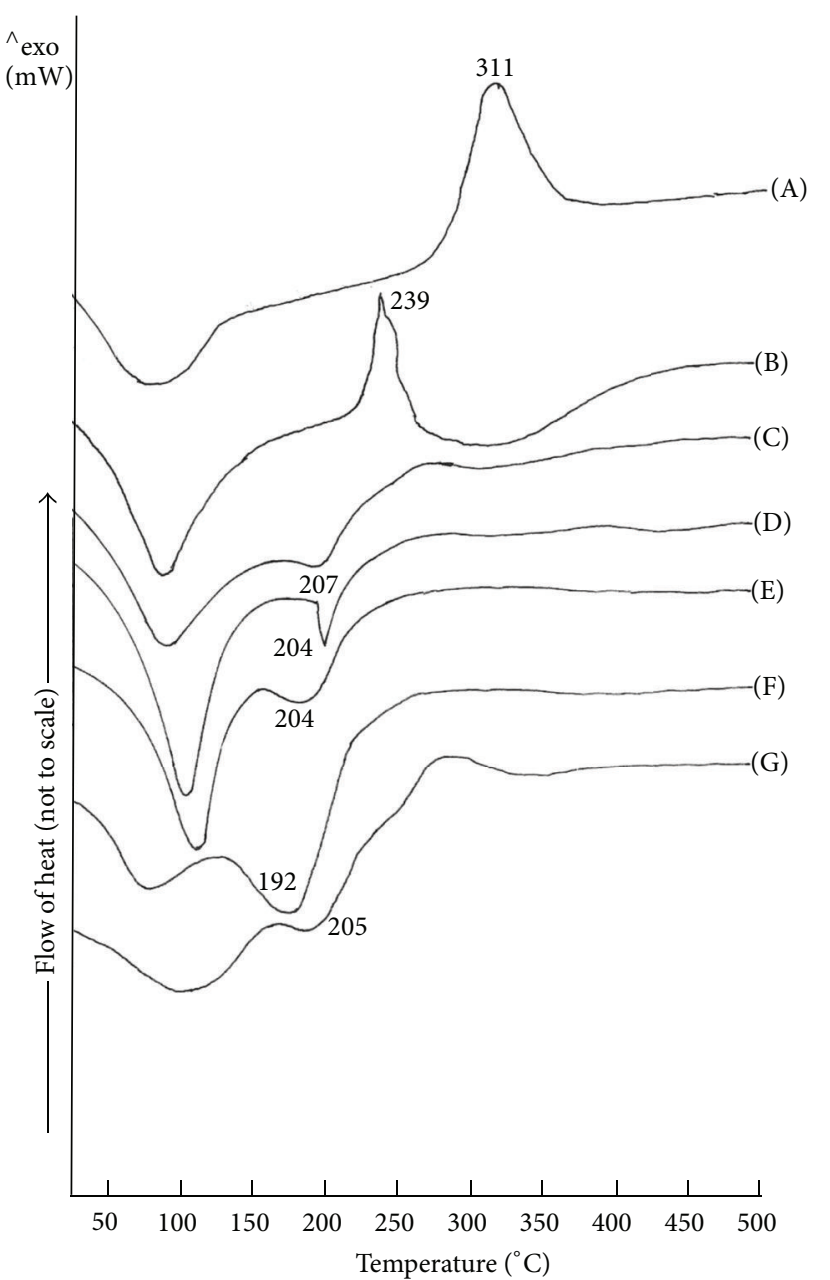

FIGURE 2: DSC thermograms of $\mathrm{CH}(\mathrm{A}), \mathrm{CS}(\mathrm{B})$, and their IPCs in the ratios of $70: 30(\mathrm{C}), 60: 40(\mathrm{D}), 50: 50(\mathrm{E}), 40: 60(\mathrm{~F})$, and $30: 70$ (G).

the second endothermic transition could be associated with the interaction between $\mathrm{CH}$ and CS during formation of IPC.

Table 1 shows the viscosity of the supernatants obtained after mixing solutions of $\mathrm{CH}$ with $\mathrm{CS}$ solutions in different ratios. CS bears a negative charge owing to the presence of $-\mathrm{COO}^{-}$and $-\mathrm{OSO}_{3}{ }^{-}$groups. $\mathrm{CH}$ bears a net positive charge due to presence of $-\mathrm{NH}_{3}{ }^{+}$groups. As a result, both polymers undergo spontaneous reaction when mixed together, resulting in formation of a solid mass. The viscosity of the supernatant obtained after mixing aqueous solutions of $\mathrm{CH}$ and $\mathrm{CS}$ was observed to increase as the proportion of $\mathrm{CH}$ in $\mathrm{CH}-\mathrm{CS}$ mixtures increased (F4, F5). However, it was observed that the viscosity decreased to a value of 1.0 when $\mathrm{CH}$ and $\mathrm{CS}$ were admixed in equal proportions. Earlier studies carried out by Tapia et al. reported that the viscosity of supernatant obtained by admixing solutions of chitosan and carrageenan drops to almost unity when the proportion of chitosan was between 30 and $40 \%$ w/w. This was ascribed to the maximum interaction between chitosan and carrageenan from $30: 70$ to $40: 60$ ratios [17]. Hence, the 


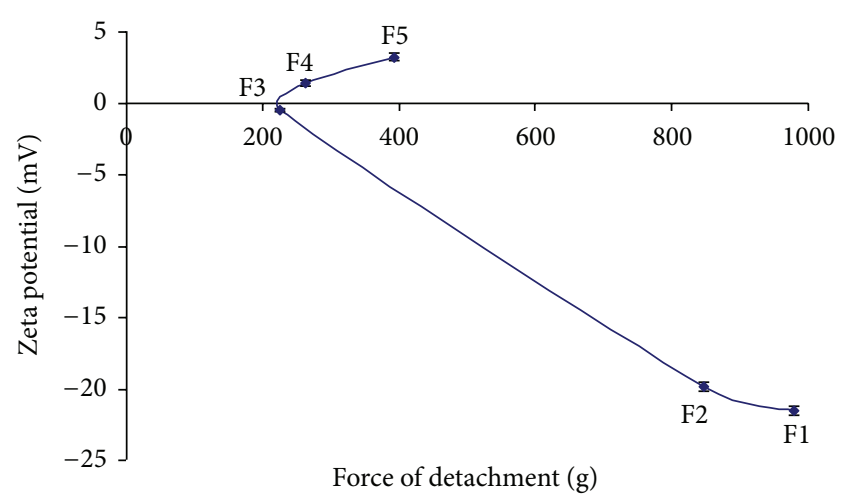

FIGURE 3: Relationship between force required for detachment and zeta potential.

present observation suggests maximum interaction between $\mathrm{CH}$ and $\mathrm{CS}$ at $50: 50$ ratio.

The result of zeta potential values employing the dispersions of the $\mathrm{CH}$ and CS polymers alone and various ratios of the IPC is depicted in Figure 3. The IPC comprising 50:50 ratio of $\mathrm{CH}$ : $\mathrm{CS}$ showed almost negligible charges and this could be attributed to maximum interaction between $-\mathrm{COO}^{-}$ and $-\mathrm{OSO}_{3}{ }^{-}$groups of $\mathrm{CS}$ with $-\mathrm{NH}_{3}{ }^{+}$group of $\mathrm{CH}$. The maximum value was of zeta potential was observed at a $\mathrm{CH}$ : CS ratio of $30: 70$. A slight positive value of zeta potential was reported for $\mathrm{CH}$ : CS; $60: 40,70: 30$ (Figure 3).

\subsection{Evaluation of Nasal Inserts}

3.2.1. Water Uptake Studies. All the nasal inserts hydrated over a period of $8 \mathrm{~h}$. The water uptake ability of all the nasal inserts is summarized in Table 1. The formulations comprising higher concentrations of $\mathrm{CH}$ (F4, F5; CH:CS $60: 40,70: 30$, resp.) showed higher water uptake ability $(P<$ $0.05)$ as compared to inserts comprising low concentrations of $\mathrm{CH}$ (F1, F2; $\mathrm{CH}: \mathrm{CS} 30: 70,40: 60$, resp.). Swelling is a function of the presence of ionized functional groups. The nasal insert prepared employing 50:50 ratio of $\mathrm{CH}$ : CS IPC (F3) was showing minimum water uptake since there was least number of ionized groups as result of maximum interaction [18]. The amine groups of $\mathrm{CH}$ are dissociated at $\mathrm{pH} 5.5$; as a result high water uptake is observed in formulations comprising high concentrations of $\mathrm{CH}$ [19].

\subsubsection{Measurement of Bioadhesive Strength. Bioadhesive} strength studies on investigational formulations were carried out using texture analyzer. The formulation F1 comprising 30:70 CH-CS IPC demonstrated the highest bioadhesive strength (maximum force required for detachment). These inserts were also depicting the highest value in zeta potential measurement indicating maximum number of ionized ions present in insert. The presence of free ionic groups in the polymeric matrix enables the polymer to interact with the functional groups present on the sialic acid residue of mucin. Further, polyanions (CS) have been reported to possess better bioadhesive strength as compared to polycations $(\mathrm{CH})$ due to greater electrostatic interactions, hydrogen bonding, hydrophobic interaction, and interdiffusion because of similar chemical structures of the adhering components [20, 21]. Figure 3 depicts the relationship between force required for detachment of insert and zeta potential. An increase in the negative zeta potential has been reported to result in an increase in bioadhesion [22]. The IPC formed between $\mathrm{CH}$ and CS were demonstrating good bioadhesion, and this is likely to be due to the increased flexibility of the $\mathrm{CH}$-CS IPC and polar functional groups. As a result of this, they are able to interact with the mucus chains and demonstrate good mucoadhesive strength, due to enhanced anchoring of the flexible chains with mucosa. Similar results have been reported by studies of chitosan/alginate hydrogels where it was found that the bioadhesive strength improved by tethering of long flexible chains to the particle surface. The resulting modified hydrogels exhibited increased mucoadhesive properties due to enhanced anchoring of the flexible chains with the mucosa [23].

3.2.3. Scanning Electron Microscopy (SEM). Figures 4(a) and 4(b) show the morphology of the nasal inserts observed by scanning electron microscopy (SEM). The structure of the nasal inserts depends on the composition of $\mathrm{CH}-\mathrm{CS}$ complexes. The process of freeze-drying is based on sublimation of the frozen water leading to the formation of pores or channels in the polymer. It was observed that $\mathrm{CH}$ (polycation) upon interaction with CS (polyanion) forms a physically cross-linked hydrogel. These hydrogels can retain a great amount of water at the interior. All the samples were characterized by a sponge-like structure [13]. Although the presence of higher amount of $\mathrm{CH}(\mathrm{F} 4, \mathrm{~F} 5)$ provided the formation of a three-dimensional interconnected porous network structure with larger and more homogenous porosity with respect to higher amounts of CS, yet all the formulations seemed to possess suitable morphology as hydrogels for nasal administration due to their more evident sponge-like structure, which can provide great insert hydration and modulation of drug release in the nasal cavity. The SEM study showed that the presence of zolmitriptan in nasal insert produced a rough surface rather than a smooth one as unloaded samples and the porous structure tend to disappear.

3.2.4. Drug Release Profiles. In vitro release studies were conducted using dissolution Apparatus-1-Basket type and phosphate buffer pH 5.5 was used as dissolution medium. The in vitro release profiles of zolmitriptan from drug loaded nasal inserts F6-F15 are depicted in Figure 5. Formulation F10 ( $\mathrm{CH}: \mathrm{CS} ; 50: 50)$ showed maximum drug release $(98.56 \%)$ in $8 \mathrm{~h}$. An increase in the $\mathrm{CH}$ concentration from $60 \%$ to $70 \%$ (formulations F12, F14; CH : CS $60: 40,70: 30$ ) resulted in a slow drug release (50\% and $30 \%$, resp., in $8 \mathrm{~h}$ ). This slow drug release could be attributed to higher water uptake (swelling) of these formulations (3.98 and 4.52, resp.). Greater swelling of the polymers has been reported to result in an increase in the diffusional path length. Thus, the drug has to diffuse through the thick viscous gel layer formed outside the polymeric matrix hence resulting in slower drug release 


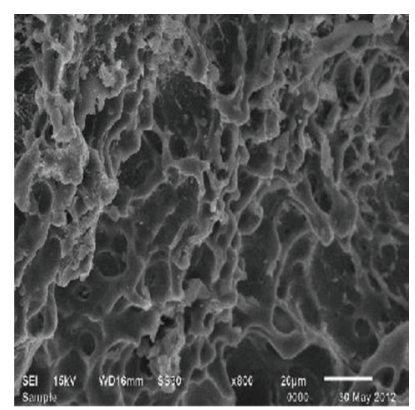

(A)

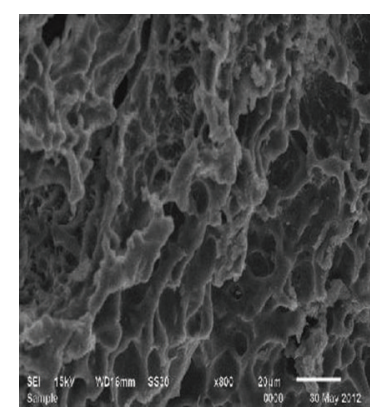

(B)

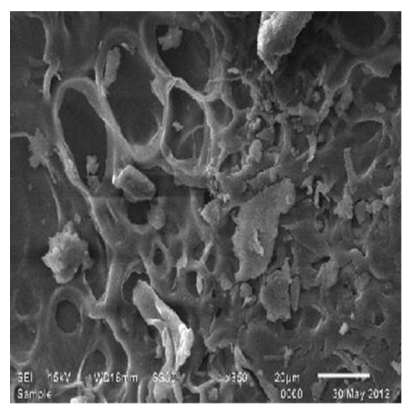

(C)

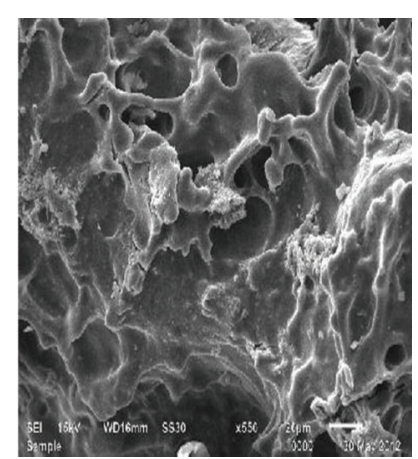

(D)

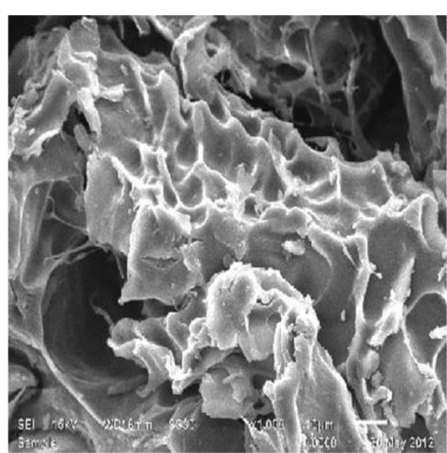

(E)

(a)

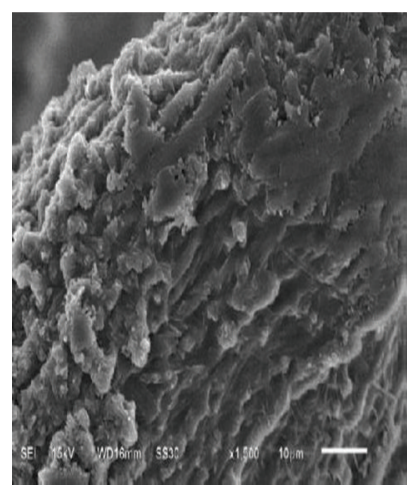

(A)

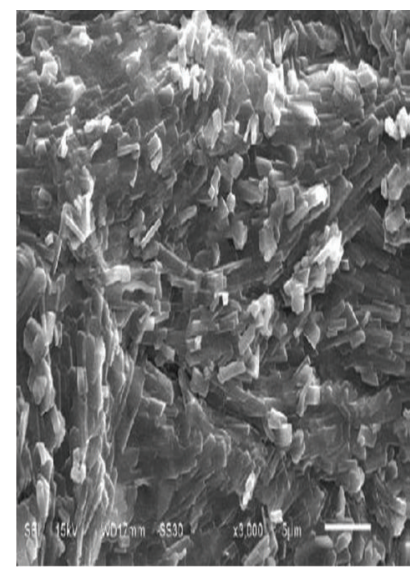

(D)

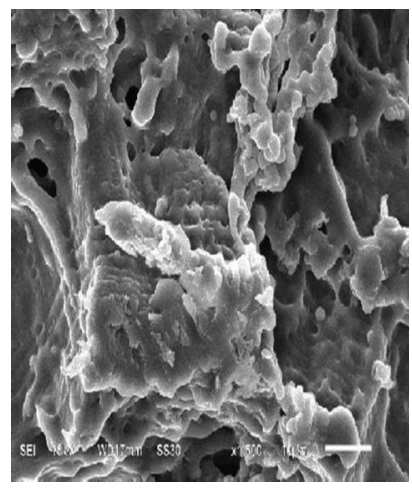

(B)

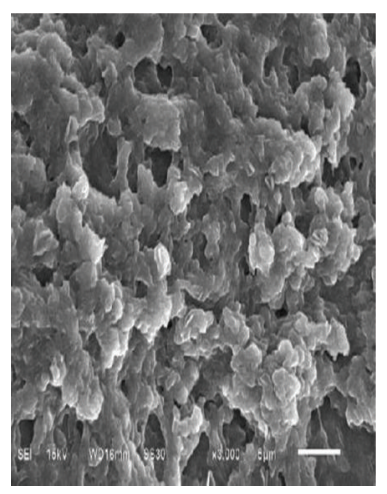

(C)

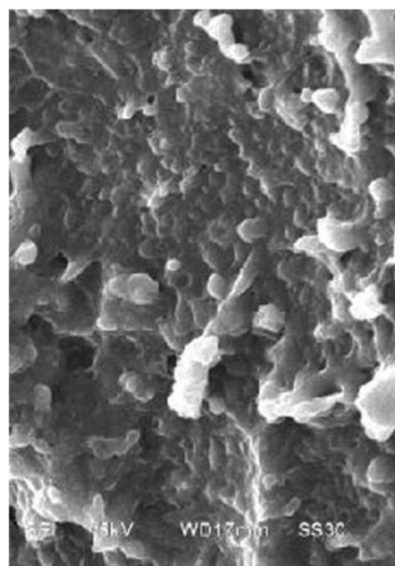

(E)

(b)

Figure 4: (a) SEM images of unloaded nasal insert: F1 (A), F2 (B), F3 (C), F4 (D), and F5 (E). (b) SEM images of loaded nasal inserts: F6 (A), F8 (B), F10 (C), F12 (D), and F14 (E). 


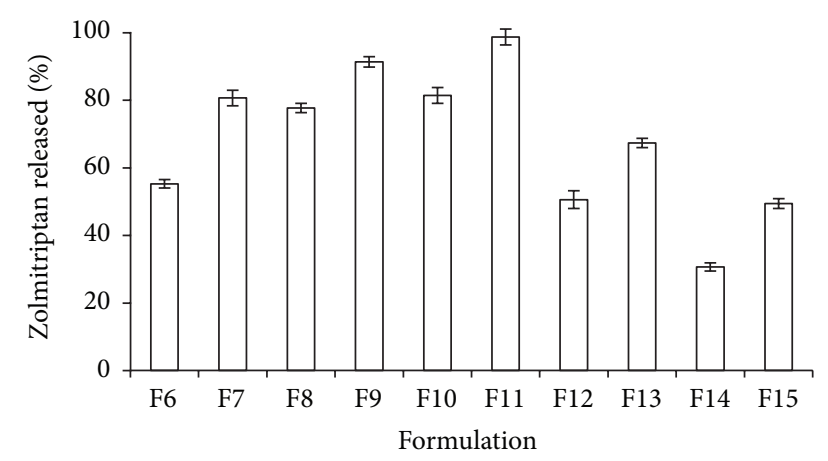

FIGURE 5: In vitro release of zolmitriptan from formulated nasal inserts.

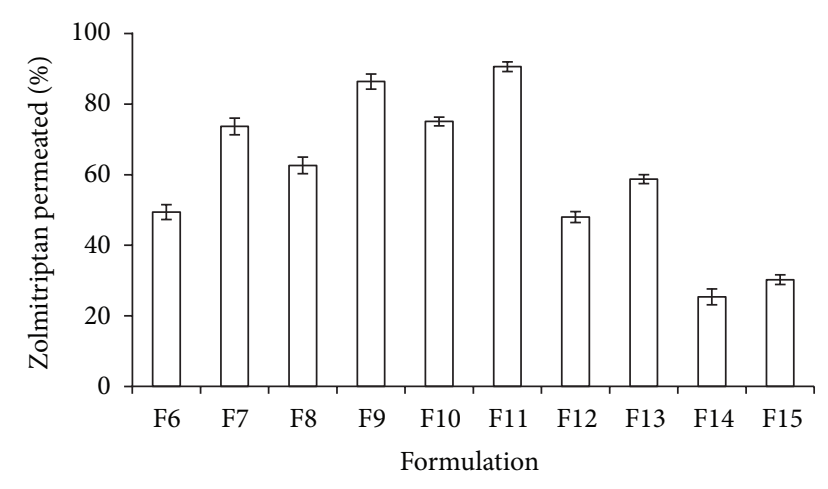

FIGURE 6: In situ permeation of zolmitriptan from formulated nasal inserts.

or release over prolonged period of time [24]. The nasal inserts were releasing statistically significant higher amounts of zolmitriptan from formulations containing drug-polymer ratio 1:10 as compared to 1:20 ratio of drug:polymer. In situ drug permeation studies also indicated that high amounts of zolmitriptan were permeating from formulations F9 and F11 ( $86 \%$ and $90 \%$, resp.) as depicted in Figure 6. However, the force required for detachment was considerably high in F9 $(845 \pm 21 \mathrm{~g})$ as compared to F11 $(245 \pm 15 \mathrm{~g})$ thus indicating F9 to be a potential candidate for delivering zolmitriptan directly to brain.

\section{Conclusion}

This investigation demonstrated that CH-CS IPC can be employed for the formulation of nasal inserts for delivery of drugs to brain. The nasal inserts were displaying porous structure and good bioadhesive strength to overcome the nasal mucociliary clearance. Further intranasal absorption studies in animals are required to confirm delivery of zolmitriptan to brain.

\section{References}

[1] S. Gupta, S. Mehrotra, C. M. Villalón, M. Perusquía, P. R. Saxena, and A. MaassenVanDenBrink, "Potential role of female sex hormones in the pathophysiology of migraine," Pharmacology and Therapeutics, vol. 113, no. 2, pp. 321-340, 2007.
[2] H. U. Sheikh and P. G. Mathew, "Acute and preventive treatment of migraine attacks," Techniques in Regional Anesthesia and Pain Management, vol. 16, no. 1, pp. 19-24, 2012.

[3] S. K. Aurora, S. H. Kori, P. Barrodale, S. A. McDonald, and D. Haseley, "Gastric stasis in migraine: more than just a paroxysmal abnormality during a migraine attack," Headache, vol. 46, no. 1, pp. 57-63, 2006.

[4] Y. Chugh, P. Kapoor, and A. K. Kapoor, "Intranasal drug delivery: a novel approach," Indian Journal of Otolaryngology and Head and Neck Surgery, vol. 61, no. 2, pp. 90-94, 2009.

[5] E. Touitou and L. Illum, "Nasal drug delivery," Drug Delivery and Translational Research, vol. 3, no. 1, pp. 1-3, 2013.

[6] U. Bertram and R. Bodmeier, "Parameters affecting the drug release from in situ gelling nasal inserts," European Journal of Pharmaceutics and Biopharmaceutics, vol. 63, no. 3, pp. 310-319, 2006.

[7] R. C. Glen, G. R. Martin, A. P. Hill et al., "Computer-aided design and synthesis of 5-substituted tryptamines and their pharmacology at the 5-HT1D receptor: discovery of compounds with potential anti-migraine properties," Journal of Medicinal Chemistry, vol. 38, no. 18, pp. 3566-3580, 1995.

[8] A. M. Ropoport, M. E. Bigal, S. J. Tepper, and F. D. Sheftell, "Zolmitriptan (Zomig)," Expert Review of Neurotherapeutics, vol. 4, no. 1, pp. 33-41, 2004.

[9] S. S. Bhattacharya, S. Shukla, S. Banerjee et al., "Tailored IPN hydrogel bead of sodium carboxymethyl cellulose and sodium carboxymethyl xanthan gum for controlled delivery of diclofenac sodium," Polymer-Plastics Technology Engineering, vol. 52, no. 8, pp. 795-805, 2013.

[10] M. Thanou, J. C. Verhoef, and H. E. Junginger, "Oral drug absorption enhancement by chitosan and its derivatives," Advanced Drug Delivery Reviews, vol. 52, no. 2, pp. 117-126, 2001.

[11] A. Bernkop-Schnürch and S. Dünnhaupt, "Chitosan-based drug delivery systems," European Journal of Pharmaceutics and Biopharmaceutics, vol. 81, no. 3, pp. 463-469, 2012.

[12] W.-B. Chen, L.-F. Wang, J.-S. Chen, and S.-Y. Fan, "Characterization of polyelectrolyte complexes between chondroitin sulfate and chitosan in the solid state," Journal of Biomedical Materials Research - Part A, vol. 75, no. 1, pp. 128-137, 2005.

[13] B. Luppi, F. Bigucci, A. Abruzzo, G. Corace, T. Cerchiara, and V. Zecchi, "Freeze-dried chitosan/pectin nasal inserts for antipsychotic drug delivery," European Journal of Pharmaceutics and Biopharmaceutics, vol. 75, no. 3, pp. 381-387, 2010.

[14] R. M. Gilhotra and D. N. Mishra, "Alginate-chitosan film for ocular drug delivery: effect of surface cross-linking on film properties and characterization," Pharmazie, vol. 63, no. 8, pp. 576-579, 2008

[15] G. Kaur, V. Rana, S. Jain, and A. K. Tiwary, "Colon delivery of budesonide: evaluation of chitosan-chondroitin sulfate interpolymer complex," AAPS PharmSciTech, vol. 11, no. 1, pp. 36-45, 2010.

[16] W. Kemp, "Infrared spectroscopy," in Organic Spectroscopy, MacMillan, London, UK, 1991.

[17] C. Tapia, Z. Escobar, E. Costa et al., "Comparative studies on polyelectrolyte complexes and mixtures of chitosan-alginate and chitosan-carrageenan as prolonged diltiazem clorhydrate release systems," European Journal of Pharmaceutics and Biopharmaceutics, vol. 57, no. 1, pp. 65-75, 2004.

[18] M. Jindal, V. Kumar, V. Rana et al., "Physico-chemical, mechanical and electrical performance of bael fruit gum-chitosan IPN films," Food Hydrocolloids, vol. 30, no. 1, pp. 192-199, 2013. 
[19] G. M. Spinks, C. K. Lee, G. G. Wallace, S. I. Kim, and S. J. Kim, "Swelling behavior of chitosan hydrogels in ionic liquid-water binary systems," Langmuir, vol. 22, no. 22, pp. 9375-9379, 2006.

[20] N. A. Peppas and P. A. Buri, "Surface, interfacial and molecular aspects of polymer bioadhesion on soft tissues," Journal of Controlled Release, vol. 2, pp. 257-275, 1985.

[21] S.-H. S. Leung and J. R. Robinson, "The contribution of anionic polymer structural features to mucoadhesion," Journal of Controlled Release, vol. 5, no. 3, pp. 223-231, 1988.

[22] K. M. Tur and H.-S. Ch'ng, "Evaluation of possible mechanism(s) of bioadhesion," International Journal of Pharmaceutics, vol. 160, no. 1, pp. 61-74, 1998.

[23] S. Wittaya-Areekul, J. Kruenate, and C. Prahsarn, "Preparation and in vitro evaluation of mucoadhesive properties of alginate/chitosan microparticles containing prednisolone," International Journal of Pharmaceutics, vol. 312, no. 1-2, pp. 113118, 2006.

[24] J. Sujja-Areevath, D. L. Munday, P. J. Cox, and K. A. Khan, "Relationship between swelling, erosion and drug release in hydrophillic natural gum mini-matrix formulations," European Journal of Pharmaceutical Sciences, vol. 6, no. 3, pp. 207-217, 1998. 

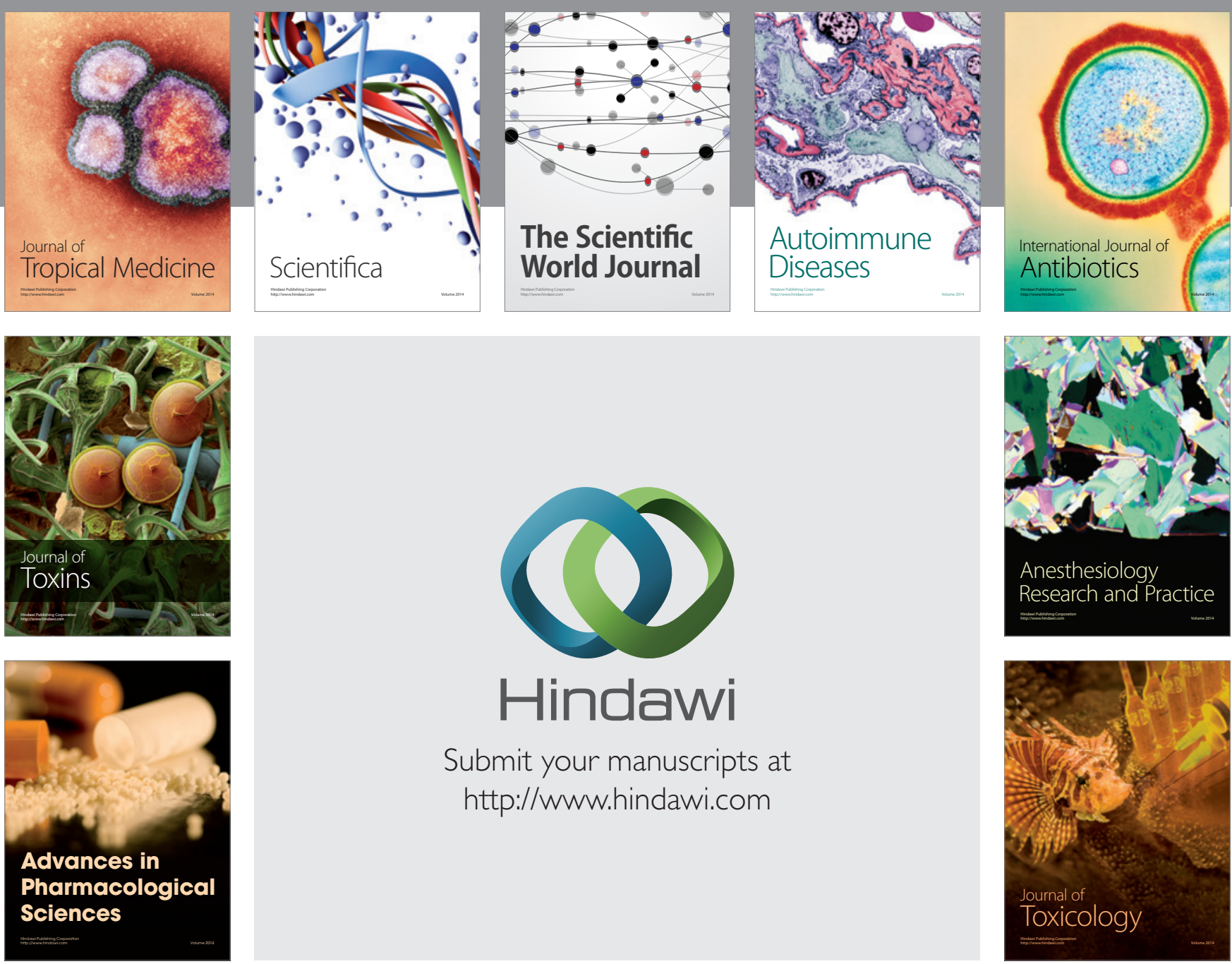

\section{Hindawi}

Submit your manuscripts at

http://www.hindawi.com
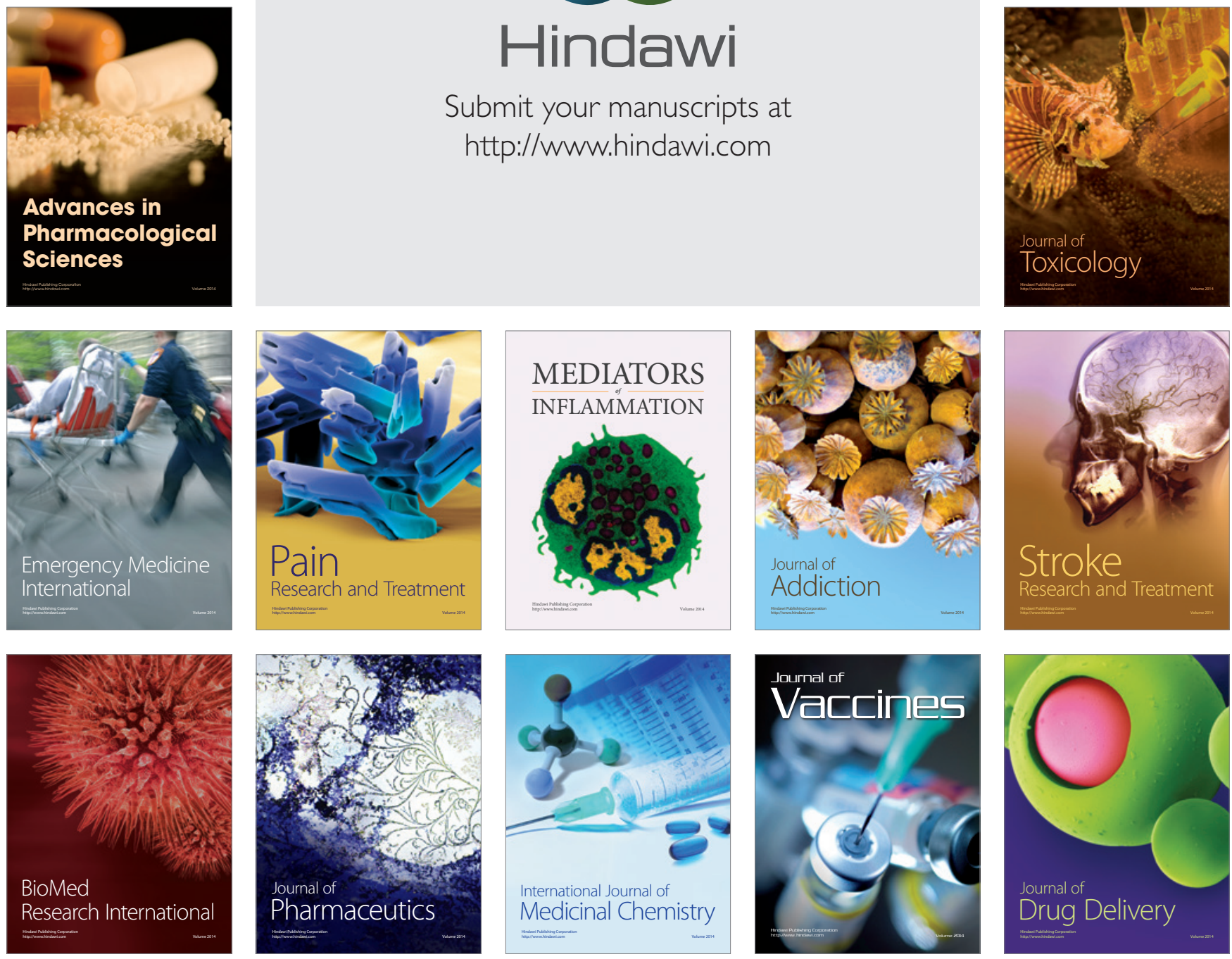